Check for updates

Cite this: RSC Adv., 2017, 7, 30533

\section{Non-stoichiometric SnS microspheres with highly enhanced photoreduction efficiency for $\mathrm{Cr}(\mathrm{vI})$ ions $\uparrow$}

\author{
Ali Rauf, (D) a Md. Selim Arif Sher Shah, (D) a Jun Young Lee, ${ }^{a}$ Chan-Hwa Chung, ${ }^{a}$ \\ Jong Wook Bae and Pil J. Yoo (D)*ab
}

Self-doped semiconductors have recently attracted extensive interest as highly efficient photocatalytic materials because the electronic properties can be modulated without sacrificing the chemical stability of the parent material. In this report, we report the facile synthesis of $\mathrm{Sn}^{2+}$ self-doped $\mathrm{SnS}$ microparticles via a simple template-free hydrothermal route. Because of the ability to successfully tune the band structure while minimizing defect generation, self-doped SnS could potentially serve as an efficient photocatalyst of wastewater. Here, $\mathrm{Sn}^{2+}$ self-doping results in insertion of an energy level slightly below the conduction band of SnS, thereby decreasing the photoexcitation energy. Furthermore, dopant sites can act as charge trapping sites, which can consequently minimize problematic charge recombination. Synthesized materials were characterized by various spectroscopic, microscopic, and surface characterization techniques, all of which confirmed the formation of self-doped $\mathrm{SnS}$. The $\mathrm{Sn}^{2+}$ selfdoped SnS photocatalyst successfully reduced carcinogenic $\mathrm{Cr}(\mathrm{VI})$ to the water-insoluble $\mathrm{Cr}(\mathrm{II})$ form under visible light illumination. The best photocatalytic efficiency was obtained from an optimal balance between increased numbers of trapping sites leading to longer charge carrier lifetime, and decreased distance between trapping sites, favoring charge recombination. We anticipate that similar methodologies can be applied to other non-stoichiometric semiconducting photocatalysts with tunable electronic properties and enhanced photocatalytic efficiency.
Received 4th April 2017 Accepted 8th June 2017 DOI: $10.1039 / c 7 r a 03854 b$ rsc.li/rsc-advances doping synthetic scheme. External doping of a parent material may create abundant defect centres that will accelerate electron-hole recombination and cause thermal and chemical instability in the resulting doped crystals. ${ }^{5}$ In contrast, selfdoping ensures preservation of the actual crystal structure while generating relatively fewer defects, thereby efficiently enhancing final photocatalytic performance due to attenuation of the electron-hole recombination rate. ${ }^{6,7}$ In recent studies, self-doped photocatalysts such as $\mathrm{Ti}^{3+}$ doped $\mathrm{TiO}_{2} / \mathrm{g}-\mathrm{C}_{3} \mathrm{~N}_{4}, \mathrm{~V}^{4+}$, $\mathrm{Ce}^{3+}$ doped $\mathrm{BiVO}_{4} / \mathrm{CeO}_{2}$, and $\mathrm{Sn}^{2+}$ doped $\mathrm{SnO}_{2-x}$ have been investigated and shown to have highly enhanced photocatalytic performance. ${ }^{8-10}$ In addition to the above-mentioned benefits of self-doping, self-doped photocatalysts can have high redox reactivity compared to undoped samples, whereas the redox activity of externally doped photocatalysts may be lower. ${ }^{10,11}$

Although conventional photocatalysts such as $\mathrm{TiO}_{2}$ have shown promising activity, their uses have been limited to absorbing light in the UV region of the solar spectrum, which is only $4 \%$ of the entire spectrum. ${ }^{11}$ Basically, photocatalysts that can absorb light in the maximum part of the solar spectrum or with very high absorption coefficients need to be developed. In addition, photocatalysts need to be physically and chemically stable to prevent impairment of oxidation or reduction reactions under illumination in aqueous medium, which can eventually minimize the problem of electron-hole
${ }^{a}$ School of Chemical Engineering, Sungkyunkwan University (SKKU), Suwon 16419, Republic of Korea.E-mail: pjyoo@skku.edu

${ }^{b}$ SKKU Advanced Institute of Nanotechnology (SAINT), Sungkyunkwan University (SKKU), Suwon 16419, Republic of Korea

$\dagger$ Electronic supplementary information (ESI) available. See DOI: $10.1039 / \mathrm{c} 7 \mathrm{ra03854b}$ first, by introducing impurities or foreign species (i.e. external doping) to increase visible light absorbance by narrowing the band gap, and secondly, by utilizing a non-stoichiometric self- 
recombination and provide a high absorbance coefficient for the entire solar spectrum. ${ }^{12}$

Tin sulfide (SnS) is a photocatalyst with a direct band gap of $1.3 \mathrm{eV}$ in bulk, which is very close to the optimum band gap of $1.5 \mathrm{eV}$ required for efficient absorption of the solar spectrum according to the Shockley-Queisser limit. ${ }^{13,14}$ In addition, it is a well-established semiconducting material with all the abovementioned desirable properties: SnS has a very high absorption coefficient $\left(>10^{4} \mathrm{~cm}^{-1}\right)$ and high hole mobility $\left(90 \mathrm{~cm}^{2} \mathrm{~V}^{-1}\right.$ $\mathrm{s}^{-1}$ ) at room temperature. ${ }^{13-15}$ More importantly, theoretical calculations have shown that it can absorb almost the entire visNIR region of the solar spectrum..$^{16}$ These properties make SnS a strong candidate for photovoltaic and photocatalytic applications. ${ }^{14,17-19}$ At the same time, SnS is a chemically stable, naturally abundant, non-toxic, and economically-viable material. ${ }^{20}$

The residual presence of heavy metal ions in water is a serious threat: plating, chemical production, and industrial waste are considered to be responsible for this problem. ${ }^{21,22}$ Among various metal ions, solubilized hexavalent chromium, $\mathrm{Cr}(\mathrm{vI})$, is a highly carcinogenic and hazardous species. Due to its high solubility in water, it is extremely difficult to remove from effluent streams. In contrast, trivalent chromium, $\mathrm{Cr}(\mathrm{III})$ is less toxic and readily precipitates under alkaline or neutral $\mathrm{pH}$ conditions. ${ }^{21}$ According to the United States Environmental Agency, $\mathrm{Cr}(\mathrm{vI})$ is one of the 17 chemicals that pose a serious threat to human beings and it is considered 100 to 1000 times more dangerous than $\mathrm{Cr}(\mathrm{III}){ }^{23}$ Although, there have been extensive investigations on the photoreduction of $\mathrm{Cr}(\mathrm{vI})$, the utilization of self-doped catalyst is yet to be explored for its maximum effectiveness. In particular, metal ion self-doping of a photocatalyst holds a strong potential for $\mathrm{Cr}(\mathrm{vI})$ reduction because of its advantage in generating excess electrons since heavy metal ion reduction is mainly driven by photo-excited electrons rather than by holes or other free radicals. Therefore, newly injected electrons will not only increase the catalytic efficiency but also help in tuning the band gap of semiconductors.

In this study, we generated Sn-self-doped SnS microspheres as efficient photocatalysts for the reduction of $\mathrm{Cr}(\mathrm{VI})$ to $\mathrm{Cr}(\mathrm{III})$. Notably, self-doped Sn acts as a donor ion and provides high electron carrier concentrations, thereby facilitating photocatalytic-Cr(vi) reduction. ${ }^{10,24}$ Introduction of Sn dopant into SnS via non-stoichiometric synthesis helped to decrease the band gap of the parent material, which enabled doping; this is an important feature in tuning photocatalytic performance. While conventional photocatalyst synthesis generally employs a template to modulate the shape of the photocatalyst, such as a surfactant or stabilizer, the method presented in this work allows for the synthesis of surface-textured SnS microspheres without the need for a template and involves a simple singlestep hydrothermal synthesis with a yield greater than $95 \%$. Furthermore, we investigated the role of polyvinylpyrrolidone (PVP) as a shaping agent as a control. Doping concentration was also optimized for maximum reduction efficiency, which was confirmed by photoluminescence characterizations; a substantial decrease in the electron-hole recombination rate was observed at the optimum concentration. Finally, we demonstrated that $\mathrm{Sn}^{2+}$ self-doped $\mathrm{SnS}$ microspheres are efficient photocatalysts for $\mathrm{Cr}(\mathrm{vI})$ reduction.

\section{Experimental section}

\subsection{Materials}

Tin(II) chloride, thioacetamide, polyvinylpyrrolidone (PVP, MW $40000)$, potassium dichromate $\left(\mathrm{K}_{2} \mathrm{Cr}_{2} \mathrm{O}_{7}\right)$, citric acid, and diphenylcarbazide were purchased from Sigma Aldrich. Absolute ethanol was purchased from Emsure ACS ISO and acetic acid was purchased from Samchun Chemicals, Korea. All reagents were of analytical grade and used without any further purification. Deionized (DI) water with a resistance of $18.2 \mathrm{M} \Omega$ was used in all experiments.

\subsection{Synthesis procedure}

In a typical synthesis, two different solutions (hereafter A and B) were prepared. For solution A, $1 \mathrm{mmol}$ of tin(II) chloride and $1 \mathrm{~g}$ of PVP were added to a mixture of $22.5 \mathrm{~mL}$ of DI water, ethanol, and acetic acid at a $3: 1: 1$ ratio and then subjected to vigorous mixing for $20 \mathrm{~min}$. Solution B containing $1 \mathrm{mmol}$ of thioacetamide in $7.5 \mathrm{~mL}$ of DI water was prepared and ultrasonicated for $20 \mathrm{~min}$. When both solutions A and B became clear, they were mixed and then stirred for $10 \mathrm{~min}$. The resulting solution was then placed in a Teflon-lined stainless steel autoclave and heated in an oven at $180^{\circ} \mathrm{C}$ for $3 \mathrm{~h}$. Next, samples were cooled to room temperature, washed sequentially with ethanol and DI water three times each, and then dried overnight in an oven at $60^{\circ} \mathrm{C}$. The dried material was ground and then used for photocatalyst applications. Samples were named according to the feed quantity of tin chloride: thioacetamide as $1: 1 \mathrm{SnS}$, $1.1: 1 \mathrm{SnS}$, and $1.2: 1 \mathrm{SnS}$, respectively.

\subsection{Characterization}

Field emission scanning electron microscopy (FESEM, JSM$7600 \mathrm{~F}$, JEOL) was used to observe particle sizes and morphologies. The crystal structures of the synthesized heterostructures were characterized using a powder X-ray diffractometer (XRD, D8 Focus, Bruker instrument, Germany) with $\mathrm{Cu} \mathrm{K} \alpha$ radiation (1.540 $\AA$ ) in the $2 \theta$ range of $10^{\circ}$ to $75^{\circ}$ at a scan rate of $0.05^{\circ} \mathrm{s}^{-1}$. Transmission electron microscopy (TEM, JEM-3010, JEOL, Japan) was performed with an acceleration voltage of $300 \mathrm{kV}$. Xray photoelectron spectroscopy (XPS) characterization was performed (ESCA 2000 instrument, VG Microtech, UK) with Al Ka $\mathrm{X}$-rays. Binding energy values were corrected and calibrated to a C 1s peak of $284.6 \mathrm{eV}$. High-resolution peaks were deconvoluted using Gaussian-Lorentzian functions with identical fullwidth-at-half-maxima (FWHM) after Shirley background subtraction. Raman spectroscopy (Micro-Raman spectrometer system, ALPHA 300M, WITec, Germany) with an excitation wavelength of $1064 \mathrm{~nm}$ was used to investigate the phase purity of SnS and the doping effect. Brunauer-Emmett-Teller (BET) specific surface areas were evaluated on the basis of nitrogen adsorption isotherms and measured at $-196{ }^{\circ} \mathrm{C}$ using a gas adsorption apparatus (ASAP 2000, Micromeritics, USA). All samples were degassed at $180{ }^{\circ} \mathrm{C}$ before nitrogen adsorption 
measurements. BET surface area was determined using adsorption data in the relative pressure $\left(P / P_{0}\right)$ range of 0.06-0.2. Barrett-Joyner-Halenda (BJH) pore size distribution was determined from desorption data. UV-visible absorption spectra were collected from the UV-vis-NIR spectrophotometer (UV-3600, Shimadzu, Japan). For photoluminescence (PL) measurements, a PL spectrometer (Scinco, FS-2, South Korea) equipped with a $150 \mathrm{~W}$ Xenon lamp as an excitation source was used.

\subsection{Photocatalytic activity}

In a typical experiment, $20 \mathrm{~mL}$ of a $15 \mathrm{ppm}$ solution of $\mathrm{Cr}(\mathrm{vI})$ was prepared by dissolving $\mathrm{K}_{2} \mathrm{Cr}_{2} \mathrm{O}_{7}$ salt in DI water. Next, $1.56 \mathrm{mM}$ citric acid was added to the $\mathrm{Cr}(\mathrm{vI})$ solution, followed by maintaining a constant $\mathrm{pH}$ of 2 using $1 \mathrm{M} \mathrm{HCl}$ solution. The colour of the $\mathrm{Cr}(\mathrm{vI})$ solution became violet upon addition of $19.77 \mathrm{mM}$ diphenylcarbazide solution in acetone. ${ }^{21}$ After the colour change, $20 \mathrm{mg}$ of catalyst was added to the solution, and the resulting mixture was ultra-sonicated for $4 \mathrm{~min}$ followed by incubation in the dark for $30 \mathrm{~min}$ for adsorption-desorption equilibrium. A solar simulator with a Xe lamp (LS-150 Xe, Abet Technologies, USA) was used as a visible light source $(\lambda>400$ $\mathrm{nm})$. The experimental mixture was placed at a distance of 100 $\mathrm{mm}$ from light source and $3.5 \mathrm{~mL}$ of sample was taken at regular intervals. The concentration of $\mathrm{Cr}(\mathrm{vI})$ was measured with the same spectrophotometer at $\lambda=540 \mathrm{~nm}$ using the diphenylcarbazide method. ${ }^{21,25}$

\subsection{Photoelectrochemical measurement}

The electrochemical analyser (CHI6143E, CH instruments, USA) was employed to measure flat band potential using MottSchottky method. Experiment was performed in a standard three-electrode system using Pt sheet as the counter electrode, $\mathrm{Ag} / \mathrm{AgCl}$ (saturated with $\mathrm{KCl}$ ) as the reference electrode, and the synthesized catalysts as the working electrodes. A $0.5 \mathrm{M} \mathrm{Na}_{2} \mathrm{SO}_{4}$ aqueous solution with $\mathrm{pH} 7$ was used as the electrolyte. Working electrodes were prepared in the following way: a paste of ethyl cellulose $(0.01 \mathrm{~g})$ and a-terpineol $(0.1 \mathrm{~mL})$ was prepared in ethanol $(0.4 \mathrm{~mL})$. Slurry was prepared with the paste and catalyst $(0.04 \mathrm{mg})$. The slurry was then coated on a $1.5 \times 1 \mathrm{~cm}^{2}$ area of a transparent ITO electrode using the doctor blade technique. The prepared electrodes were then dried in an oven and mildly calcined at $300{ }^{\circ} \mathrm{C}$ for $60 \mathrm{~min}$ to remove ethyl cellulose and aterpineol. All the electrodes had similar film thickness (10-15 $\mu \mathrm{m})$. Mott-Schottky measurement was performed at $1 \mathrm{kHz}$ on a scan rate $50 \mathrm{mV} \mathrm{s}^{-1}$ with potential range from -0.8 to $+0.6 \mathrm{~V}$.

\section{Results and discussion}

\subsection{Mechanism of particle synthesis}

A general schematic of the synthesis process of SnS microspheres is provided in Fig. 1. FESEM observation was performed to investigate the sequence of particle growth and structural evolution at different time intervals during the hydrothermal reaction, i.e. 4, 30, 120, and $180 \mathrm{~min}$, respectively (Fig. 2a-d). As shown in Fig. 2a, microsphere formation started with the formation of nanoparticles. These nanoparticles aggregated to

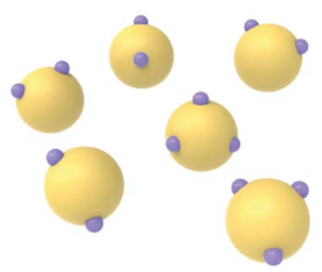

Nanoparticle formation

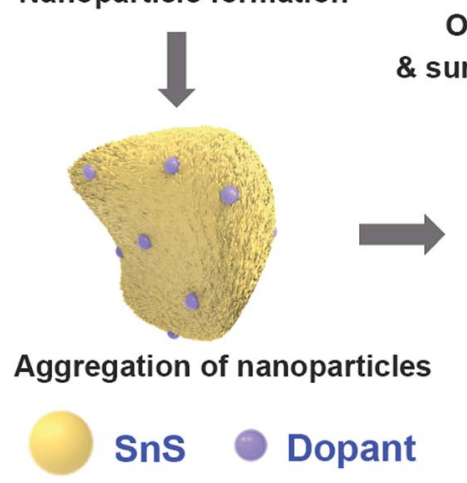

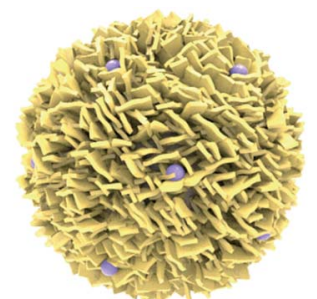

Ostwald ripening

\& surface roughening

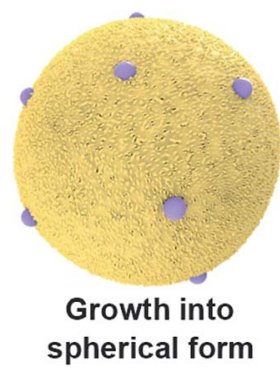

Fig. 1 Schematic illustration of synthesis of SnS microspheres.

form rough-textured microspheres by a process of oriented aggregation. Here, oriented aggregation refers to the crystal growth phenomenon where primary nanoparticles first selfassemble, then undergo crystallographic reorganization to give rise to the secondary particles of underdeveloped spheres, as captured in Fig. 2 b. ${ }^{26}$

As the reaction proceeded, particle size increased, and complete spheres were obtained, as shown in Fig. 2c. With further prolonging of the reaction, the surface morphology of particles changed because of the difference in energy between the exterior and interior of the particles; the outer region had greater surface energy and was therefore less stable than the inner part. The surface region therefore preferentially dissolved into solution and the resulting Ostwald ripening process resulted in formation of rough-textured microspheres, as shown in Fig. 2 d. $^{25,27}$ The effect of self-doping on particle morphology and shape was also investigated. Self-doping appeared to have little effect on the shape or morphology of the microparticles that were obtained, as can be seen in Fig. 2d-f. Notably, as revealed in Fig. $2 \mathrm{~g}$, the surfaces of the microspheres were completely covered with incorporated nanoplatelets. Additional experimental results performed in the absence of PVP clearly demonstrated the role of PVP as a shaping agent (see Fig. 2h). ${ }^{28}$

\subsection{Material characterization}

We investigated the crystallographic structure of tin sulfide (SnS) using XRD data (Fig. 3). The peak positions of all samples were in accordance with JCPDS 039-0354 for orthorhombic SnS. ${ }^{14}$ The $2 \theta$ peaks at 26,31 , and $51^{\circ}$ were indexed to the $(120)$, (111), and (112) planes of orthorhombic SnS. Smaller peaks corresponding to the face-centred cubic (FCC) planes of orthorhombic SnS were also observed. In particular, no peaks for other phases, such as $\mathrm{Sn}_{2} \mathrm{~S}_{3}$ or $\mathrm{SnS}_{2}$, were observed. Therefore, 

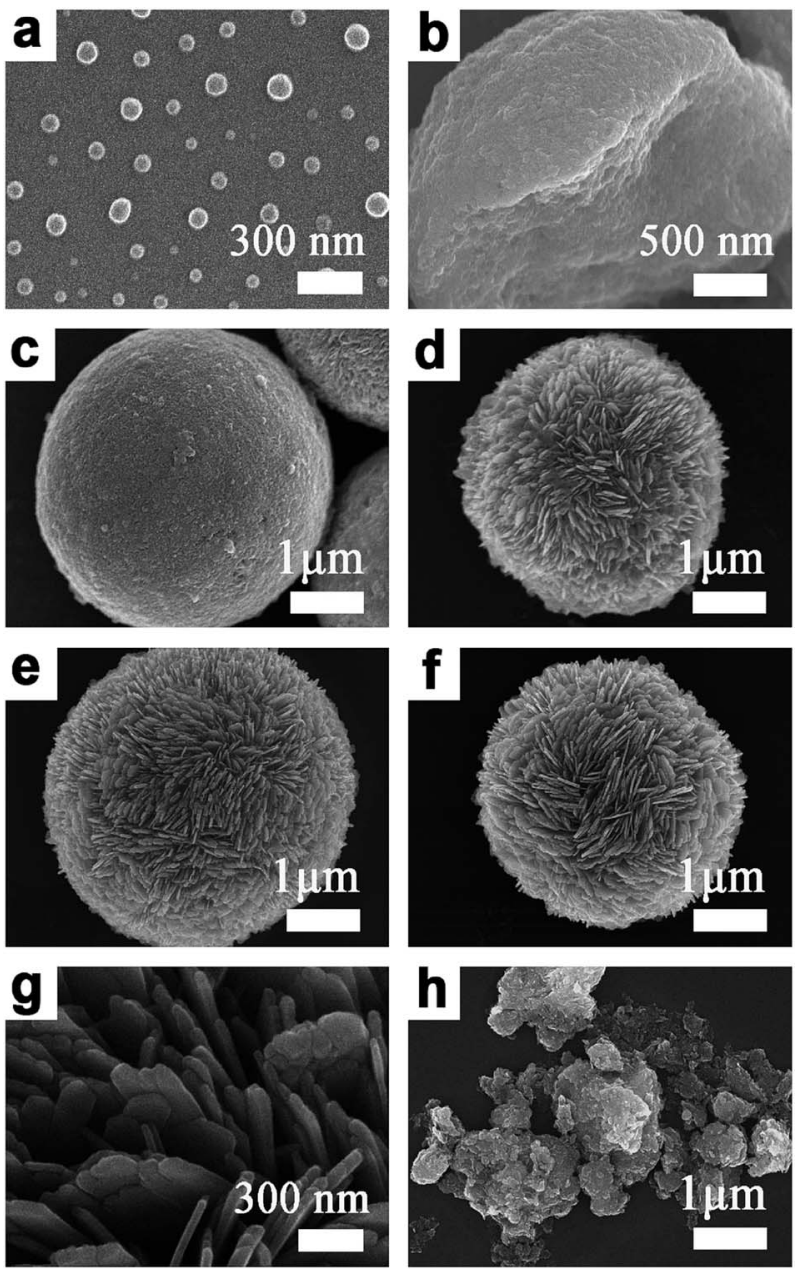

Fig. 2 FESEM images of (a) 4 min SnS (b) 30 min SnS, (c) 120 min SnS, (d) 180 min $1: 1 \mathrm{SnS}$ (e) $1.1: 1 \mathrm{SnS}$, (f) $1.2: 1 \mathrm{SnS}$, (g) high magnification SEM image of $1.1: 1 \mathrm{SnS}$ and (h) $1.1: 1 \mathrm{SnS}$ without PVP.

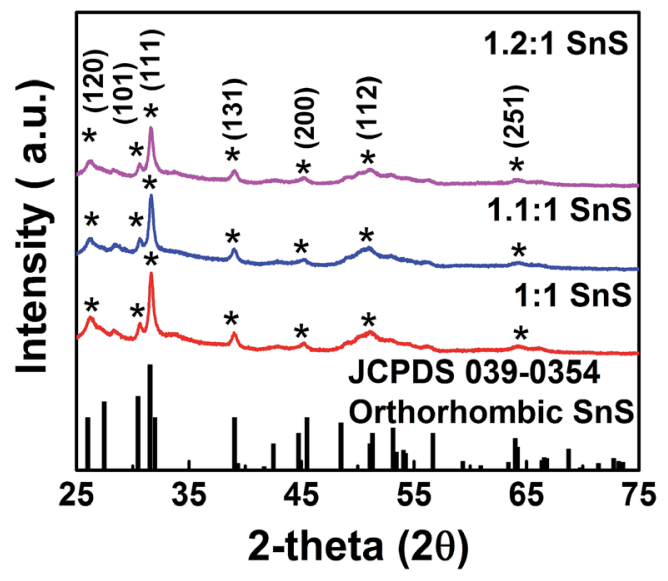

Fig. 3 XRD of all samples.

the XRD data unambiguously confirmed the formation of tin sulfide (SnS). Furthermore, for the doped samples $1.1: 1 \mathrm{SnS}$ and $1.2: 1 \mathrm{SnS}$ ), there was no change in peak positions or formation of new peaks, supporting no significant difference in crystallography of the parent material after doping. Crystallite size of all samples was calculated from (111) FCC of SnS using the Scherrer equation (Table S1 $\dagger$ ). Crystallite size decreased slightly in response to doping, although the change in crystallite size did not appear to depend on the doping level. ${ }^{\mathbf{1 0}}$

High resolution TEM observation was performed to investigate the presence of dopant sites in the tin sulfide samples. TEM images of 1.1:1 SnS are provided in Fig. 4a and b. Sn dopant sites within the matrix of SnS were clearly identifiable. Lattice spacings of $0.37 \mathrm{~nm}$ and $0.28 \mathrm{~nm}$ correspond to $\mathrm{Sn}$ (111) (JCPDS 01-086-2266) and SnS (111), respectively. The lattice spacing calculated for $\mathrm{SnS}$ is in accordance with the XRD results. To explicate the chemical state and composition of tin sulfide, XPS characterization was performed. As shown in Fig. 5a, the low energy spectrum confirmed the presence of Sn and $\mathrm{S}$ in the photocatalyst material. The high-resolution spectrum of Sn 3d (Fig. 5b) exhibited two main doublet spin orbit peaks at $486 \mathrm{eV}$ and $494 \mathrm{eV}$ corresponding to $\mathrm{Sn}^{2+} 3 \mathrm{~d}_{5 / 2}$ and $\mathrm{Sn}^{2+}$ $3 \mathrm{~d}_{3 / 2}$, respectively. ${ }^{29,30}$ Furthermore, the Sn $3 \mathrm{~d}$ peaks were slightly shifted to a lower energy, which confirmed $\mathrm{Sn}^{2+}$ doping (Fig. S1†). ${ }^{31}$ Similarly, as shown in Fig. 5c, S 2p showed two peaks at binding energies of $160.9 \mathrm{eV}$ and $161.9 \mathrm{eV}$. Valence band XPS spectra (VB-XPS) near the Fermi level were obtained to investigate the electronic structure of doped SnS (Fig. 5d); the valence band edge for 1.1 : $1 \mathrm{SnS}$ was $1.43 \mathrm{eV}$.

Next, BET surface area was measured and results are tabulated in the ESI (Fig. S2 and Table S2 $\dagger$ ). BET surface area increased slightly with doping, presumably due to doping facilitating the Ostwald ripening process. Raman spectroscopic analysis was also carried out to confirm the successful formation of SnS and the effect of $\mathrm{Sn}^{2+}$ self-dopant (Fig. 6). Three main peaks were observed at 91, 178 and $221 \mathrm{~cm}^{-1}$ for undoped SnS; the first peak corresponded to Ag mode and the other two peaks corresponded to $\mathrm{B}_{1 \mathrm{u}}$ mode. ${ }^{32,33}$ A peak shift was clearly observed for the $\mathrm{Sn}^{2+}$ doped samples, confirming successful doping with $\mathrm{Sn}^{2+}$. To calculate the band gap of the synthesized materials, the Kubelka-Munk equation was applied to UV-diffuse reflectance spectroscopy (UV-DRS) data as shown in Fig. 7 using eqn (1):

$$
F(R)=(1-R)^{2} /(2 R)=\alpha / s
$$

where $F(R)$ is the Kubelka Munk function, ' $R$ ' is reflectance, ' $\alpha$ ' is the absorption coefficient, and ' $s$ ' is the scattering coefficient. The scattering coefficient, $s$, can be ignored on the basis of
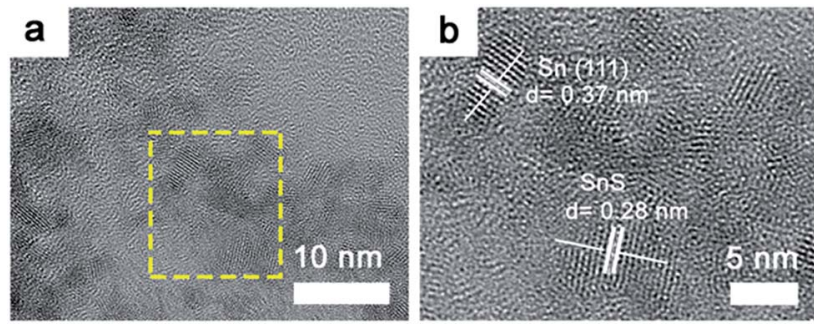

Fig. 4 (a) TEM image of $1.1: 1 \mathrm{SnS}$, and (b) high magnification TEM image of $1.1: 1 \mathrm{SnS}$. 

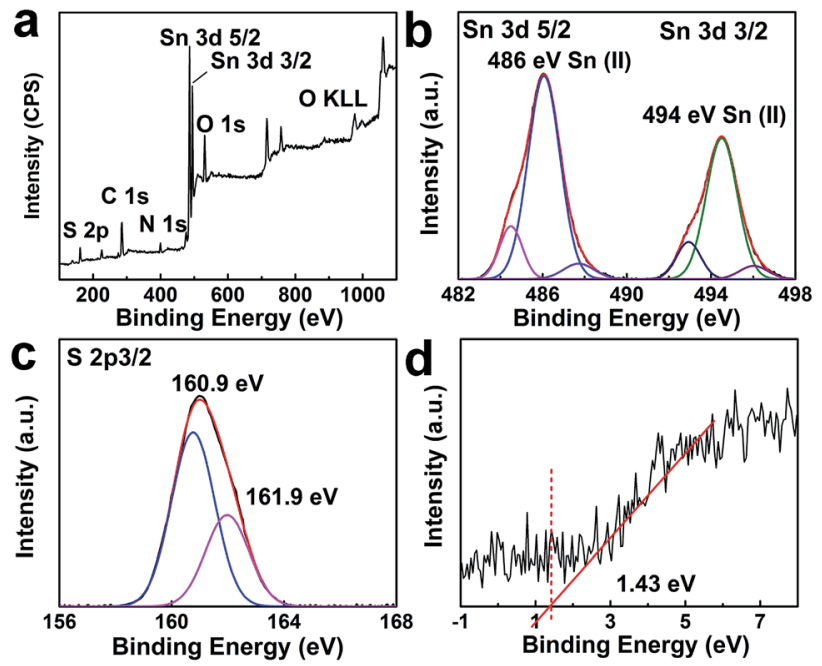

Fig. 5 (a) Survey XPS spectrum of $1.1: 1$ SnS. (b) Core level Sn 3d spectra. (c) Core level XPS spectra of $S 2 p_{3 / 2}$. (d) VB-XPS spectra of $1.1: 1 \mathrm{SnS}\left(\mathrm{Sn}^{2+}\right.$ doped).

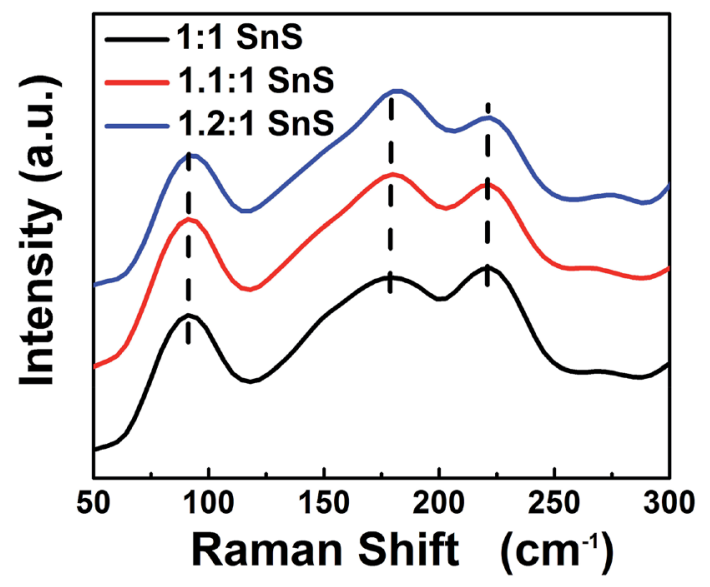

Fig. 6 Raman Spectrum of all samples.

wavelength dependence, which makes $F(R)$ proportional to $\alpha .^{34}$ Tauc, Davis and Mott proposed an equation to calculate the band gap using the absorption coefficient as follows: ${ }^{35,36}$

$$
(h \nu \alpha)^{1 / n}=A\left(h \nu-E_{\mathrm{g}}\right)
$$

Here, $n=1 / 2$ for direct band semiconductors and $n=2$ for indirect band semiconductors. $h$ is Planck's constant, $\nu$ is vibration frequency, and $E_{\mathrm{g}}$ is band gap. By substituting $n=1 / 2$ and $\alpha=F(R)$ into eqn (2), the band gap was calculated by extrapolation of $(h \nu \alpha)^{2} v s$. $h \nu$, as shown in Fig. 7b. The estimated band gaps of the synthesized materials (Table 1) were close to the reported values (Table 2). Furthermore, the valence band potential and the conduction band potential were calculated from valence band spectra (VB-XPS) and $E_{\mathrm{g}}$. With $\mathrm{Sn}^{2+}$ doping, there was a prominent change in the conduction band position. This may have originated from the formation of a discrete

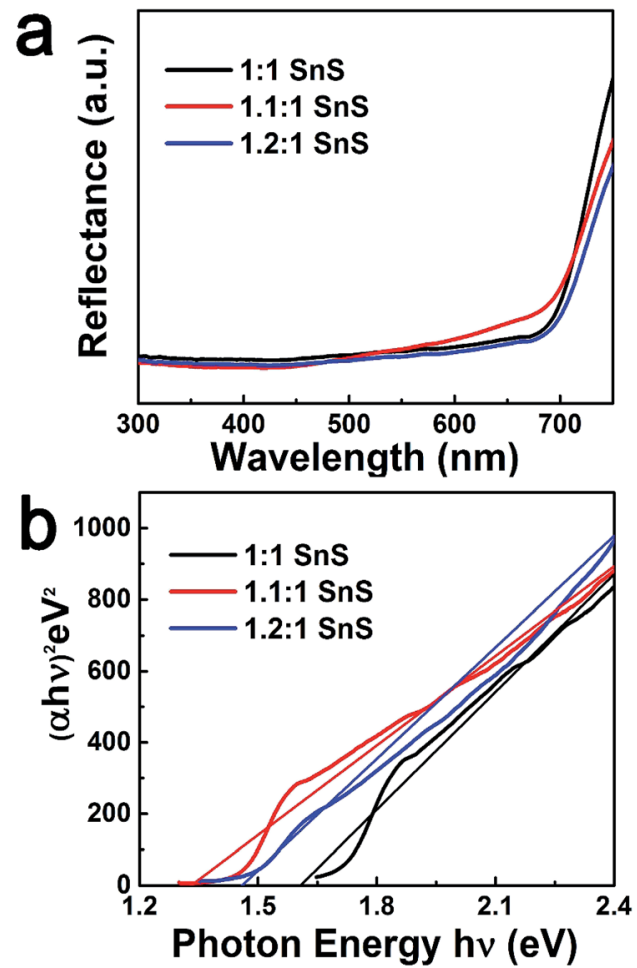

Fig. 7 UV-DRS spectrum according to Kubelka-Munk equation of all samples.

energy state below the conduction band that decreased the photonic excitation energy required for charge carrier transition. ${ }^{24}$ Accordingly, the band gap would have decreased, explaining why $\mathrm{Sn}^{2+}$ doping improved photocatalytic performance. However, when the $\mathrm{Sn}^{2+}$ dopant concentration further increased, the band gap increased slightly, though it was still lower than that of the parent un-doped SnS. A plausible reason for this observation is the Burstein-most effect, which states that the Fermi level shifts above the conduction band once the density of states in the conduction bands are populated. ${ }^{37}$

\subsection{Photocatalytic performance}

To investigate the photocatalytic performance of synthesized samples, $\mathrm{Cr}(\mathrm{vI})$ reduction experiments were performed for $\mathrm{Cr}(\mathrm{vI})$ solution at a concentration of $15 \mathrm{ppm}$ using a solar light simulator as the light source. Fig. 8a shows the photoreduction of $\mathrm{Cr}(\mathrm{vI})$ with different photocatalysts under ambient temperature and pressure. For comparison, conventional $\mathrm{TiO}_{2}$ nanoparticles of P25, N-doped $\mathrm{TiO}_{2}$, and $1: 1 \mathrm{SnS}$ (undoped $\mathrm{SnS}$ ) were used as reference photocatalyst materials. Citric acid was used as the hole scavenger species. ${ }^{25} C_{0}$ is the concentration of $\mathrm{Cr}(\mathrm{vI})$ after reaching equilibrium in the dark. As can be seen in in Fig. 8a, P25 and N-doped $\mathrm{TiO}_{2}$ showed very poor catalytic performance with only $13 \%$ and $20 \%$ reduction in $40 \mathrm{~min}$, respectively, whereas the catalytic efficiency of the undoped SnS sample was better than that of the other two reference materials. Efficiency of the $\mathrm{Sn}^{2+}$ self-doped samples was high; $\sim 100 \%$ reduction within 30 min of irradiation. 
Table 1 Estimated band gap, conduction band (CB), and valence band (VB) positions of all samples

\begin{tabular}{llll}
\hline & $\begin{array}{l}\text { Estimated } \\
\text { band gap } \\
(\mathrm{eV})\end{array}$ & $\begin{array}{l}\text { Estimated } \\
\text { CB position } \\
\text { vs. NHE }(\mathrm{eV})\end{array}$ & $\begin{array}{l}\text { Estimated } \\
\text { VB position } \\
\text { vs. NHE }(\mathrm{eV})\end{array}$ \\
\hline $1: 1 \mathrm{SnS}$ & 1.6 & -0.19 & 1.41 \\
$1.1: 1 \mathrm{SnS}$ & 1.32 & 0.11 & 1.43 \\
$1.2: 1 \mathrm{SnS}$ & 1.45 & -0.01 & 1.44 \\
\hline
\end{tabular}

Table 2 Report band gap values (eV) of SnS

Band gap

(eV)

Reference

1.24

1.32

1.43

1.60

14

38

39

40

Therefore, $\mathrm{Sn}^{2+}$ self-doped samples showed significantly better reduction performance than undoped SnS. As can be seen in Fig. $7 \mathrm{c}$ the reaction rates increased to $0.2274 \mathrm{~min}^{-1}$ and $0.1661 \mathrm{~min}^{-1}$ for self-doped samples $(1.1: 1 \mathrm{SnS}$ and $1.2: 1 \mathrm{SnS}$, respectively), whereas the reaction rate was lower for the undoped sample $\left(0.13944 \mathrm{~min}^{-1}\right)$. Here, the increased reduction efficiency obtained for the $\mathrm{Sn}^{2+}$ self-doped $\mathrm{SnS}$ can be attributed to the dual role of the self-dopant. First, selfdoping increased the charge separation barrier for suppressing electron-hole recombination by providing charge trap sites, and secondly, self-doping decreased the band gap on the electronic scale. Doping generally creates trap sites, and their numbers increase with increasing dopant concentration, favouring photocatalytic performance while minimizing the electron-hole recombination rate. Similarly, a decrease in the band gap usually occurs because of the creation of intermediate energy states within the forbidden gap, thus decreasing the excitation energy reduction efficiency reached a maximum under an optimally adjusted self-doping concentration of $1.1: 1$ SnS. Further increases in self-dopant concentration decreased the reduction efficiency, as shown for the $1.2: 1 \mathrm{SnS}$ sample. This may be because the dopant concentration exceeded an optimum value, increasing the number of trap sites within one particle and consequently decreasing the distance between trap sites, resulting in population of charge recombination sites rather than generation of intrinsic charge trap sites. ${ }^{41} \mathrm{~A}$ second reason to explain this observation may be the change in band gap; band gap increased slightly from $1.32 \mathrm{eV}$ to $1.45 \mathrm{eV}$ for 1.2:1 SnS. Digital photographic image showing visible identification of $\mathrm{Cr}(\mathrm{VI})$ reduction to $\mathrm{Cr}(\mathrm{III})$ using a sample of the doped SnS is provided in Fig. S3. $\dagger$ To further investigate $1.1: 1 \mathrm{SnS}$ sample as the most optimal dopant concentration, two more samples with different concentrations $(1.05: 1.1 \mathrm{SnS}$ and $1.5: 1 \mathrm{SnS})$ were prepared and investigated for photocatalytic activity as shown in Fig. S4. $\dagger$ As a result, sample with
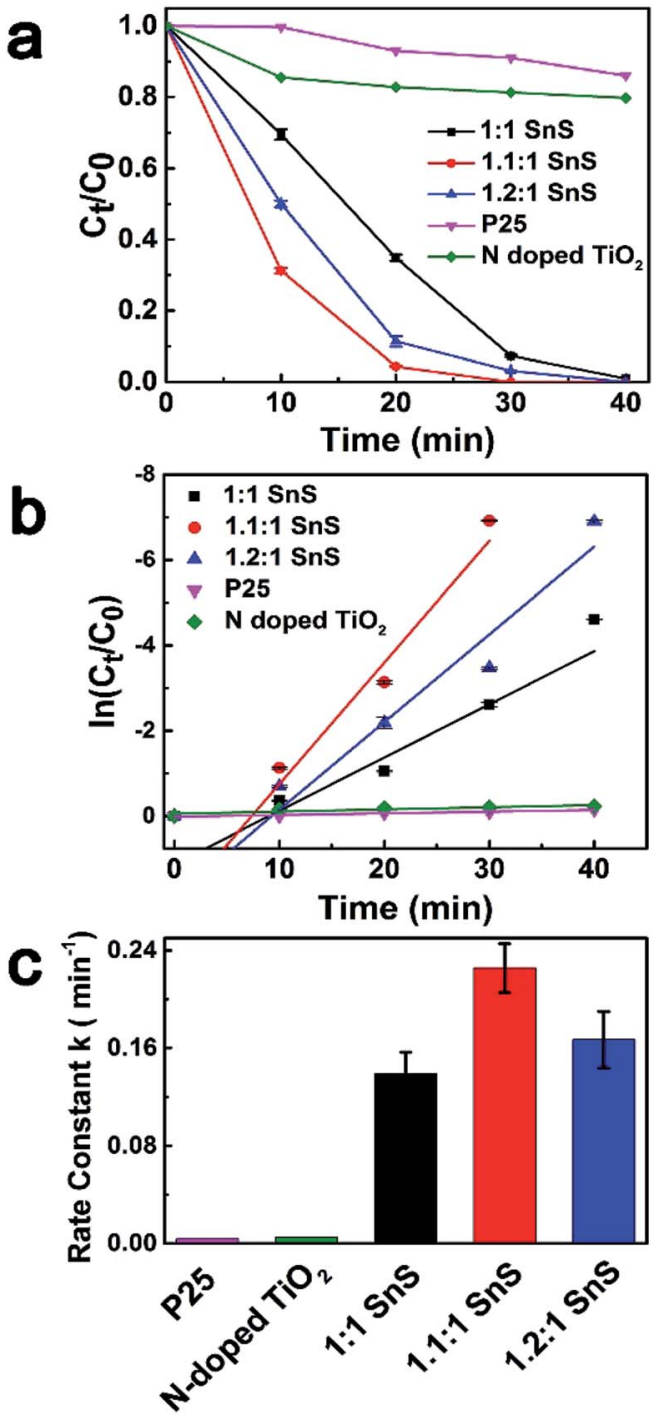

Fig. 8 Photoreduction of $\mathrm{Cr}(\mathrm{VI})$ under visible light irradiation. (a) $C_{t} / C_{0}$ concentration changes of $\mathrm{Cr}(\mathrm{VI})$ as a function of time using different catalysts. (b) Logarithmic concentration ratio of the samples as a function of time with different catalysts. (c) Bar plot showing the differences in rate constants.

$1.1: 1 \mathrm{SnS}$ showed the best performance. Recently, there has been a study using $\mathrm{Sn}^{4+}$ self-doped $\mathrm{SnS}$ for $\mathrm{Cr}(\mathrm{vI})$ reduction, wherein the ratio between $\mathrm{Cr}(\mathrm{vI})$ ion and photocatalyst amount $(0.02)$ is nearly the same with the present study. In that work, there reported the presence of $\mathrm{SnS}_{2} / \mathrm{SnS}$ heterojunctions along with doping sites, which is clearly discerned from this work. Apart from this difference, with regard to the photocatalytic activity, the present system has shown superior performances in reducing $\mathrm{Cr}(\mathrm{vI})$ ions. $^{42}$

Reduction of $\mathrm{Cr}(\mathrm{vI})$ via photocatalytic reaction is well understood and had been widely investigated. ${ }^{43,44}$ Visible light excites the electrons of the catalyst material, which eventually attack the dichromate group $\left(\mathrm{Cr}_{2} \mathrm{O}_{7}{ }^{2-}\right)$ to reduce $\mathrm{Cr}(\mathrm{VI})$ to $\mathrm{Cr}(\mathrm{III})$. Meanwhile, the holes generated as a result of this photoexcitation are consumed by citric acid, which functions as a hole 
scavenger in this system. The reaction mechanism can be explained as

$$
\begin{array}{r}
4 \mathrm{Cr}_{2} \mathrm{O}_{7}^{2-}+\mathrm{C}_{6} \mathrm{H}_{5} \mathrm{O}_{7}^{3-}+41 \mathrm{H}^{+}+6 \mathrm{e}^{-} \rightarrow \\
8 \mathrm{Cr}^{3+}+6 \mathrm{CO}_{2}+23 \mathrm{H}_{2} \mathrm{O}
\end{array}
$$

In addition, synthesized samples of doped SnS were checked for their reusability and stability. As shown in Fig. 9, photocatalytic performance was mostly retained after five cycles. The stability of self-doped SnS to irradiation was also investigated through XRD; irradiation did not affect the crystallography of the materials as shown in Fig. 10. In order to investigate further potential of the synthesized material for highly concentrated condition of $\mathrm{Cr}(\mathrm{vI}), 50 \mathrm{ppm}$ of $\mathrm{Cr}(\mathrm{vI})$ was also tested for a reduction reaction and the results are provided in Fig. S5. $\dagger$ It has shown considerably good performance with a reduction efficiency of $81 \%$ in 50 min of light irradiation.

\subsection{Photoluminescence measurements}

Electron hole recombination rate was characterized using photoluminescence, as shown in Fig. 11. The peak intensity of the emission peak in the PL spectra has a direct relation to the electron hole recombination rate. For this analysis, excitation was done at $550 \mathrm{~nm}$. A clear sharp peak emerged at $822 \mathrm{~nm}$, which is a characteristic peak of SnS. Furthermore, the position of the emission peak directly corresponded to the band gap of $\mathrm{SnS}$, i.e. $1.6 \mathrm{eV}$ as calculated from UV-vis spectrophotometry. The peak intensity varied in the same manner as the changes in photocatalytic activity. Maximum electron-hole recombination rate was observed for undoped $\mathrm{SnS}$ (1:1 SnS). With $\mathrm{Sn}^{2+}$ selfdoping, the electron-hole recombination rate was supressed, as can be seen from the lower peak intensities in the PL spectra of the self-doped samples (1.1:1 $\mathrm{SnS}$ and 1.2:1 SnS). The minimum electron-hole recombination rate was observed at the optimum self-doping concentration of 1.1:1 SnS. The minimum electron-hole recombination rate depends on the number of trap sites, as described in the previous section. A slight peak shift was observed, which may have been due to an increase in the amount of self-dopant.

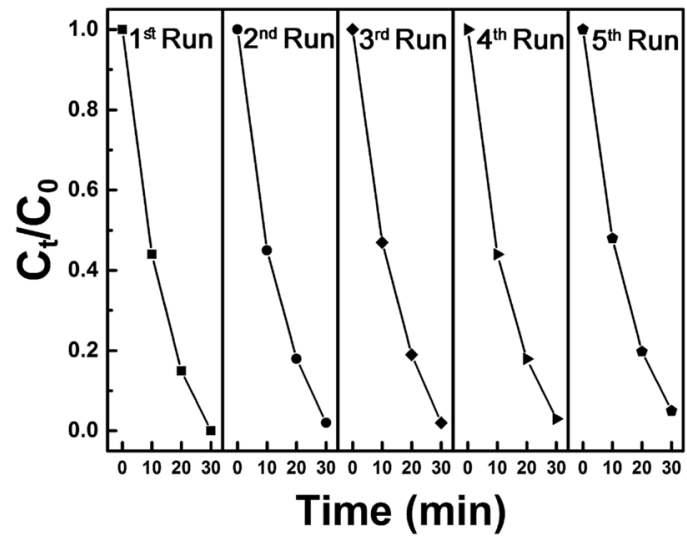

Fig. 9 Five cycles of photoreduction of $\mathrm{Cr}(\mathrm{VI})$ using $1.1: 1 \mathrm{SnS}$ as a photocatalyst under visible light irradiation for 30 minutes.

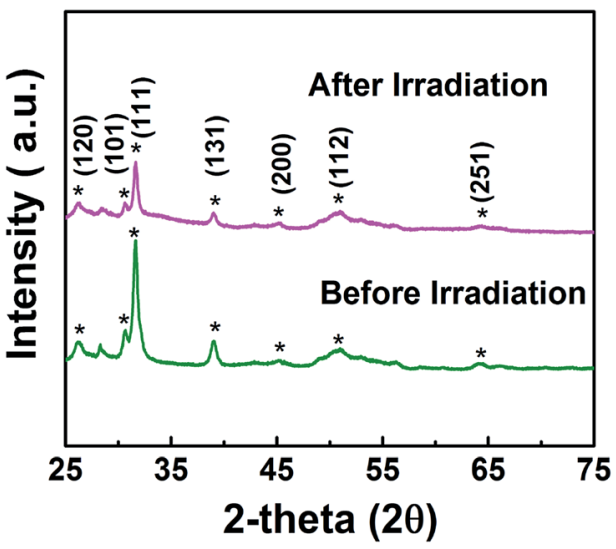

Fig. 10 XRD of 1.1:1 SnS (doped SnS) sample before and after irradiation.

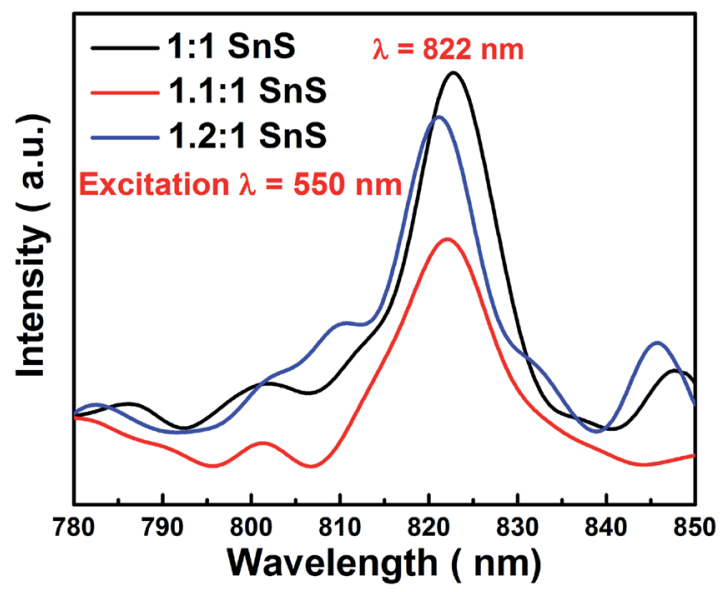

Fig. $11 \mathrm{PL}$ spectrum of different samples excited at $550 \mathrm{~nm}$.

\subsection{Energy band diagram}

Finally, to further confirm the formation of intermediate energy state in the forbidden energy gap, conduction band position was studied as a function of flat band potential. As shown in Fig. 12a, Mott-Schottky method was employed for this purpose. It can be seen that the synthesized doped and un-doped SnS typically exhibit n-type semiconductor characteristics with a positive slope. Moreover, flat band position changes according to doping treatment. It can be deduced that doping would result in a creation of intermediate energy state, which is also indicated by a change in the band gap and VB-XPS. The flat band potential is considered to be approximately equal to the conduction band potential. ${ }^{45} \mathrm{~A}$ change in the flat band potential also implies a concurrent change in the conduction band potential. For a undoped 1 : 1 $\mathrm{SnS}$ ) sample, the value of the flat band potential (VFB) is equal to $-0.79 \mathrm{~V}$, whereas in the case of self-doped $\mathrm{SnS}(1.1: 1 \mathrm{SnS}) \mathrm{VFB}$ shifts to $-0.75 \mathrm{~V}$. Hence, the formation of intermediate energy state is well verified using Mott-Schottky method.

Energy band diagrams for $\mathrm{Cr}(\mathrm{vI})$ reduction for the doped sample are presented in Fig. 12b. This highlights the role of doping in enhancing the photocatalytic activity of SnS. The 
a

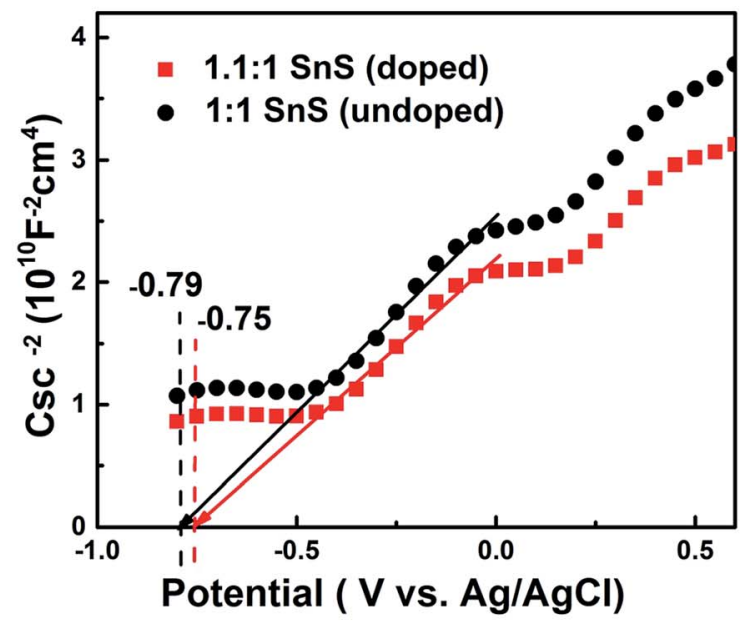

b

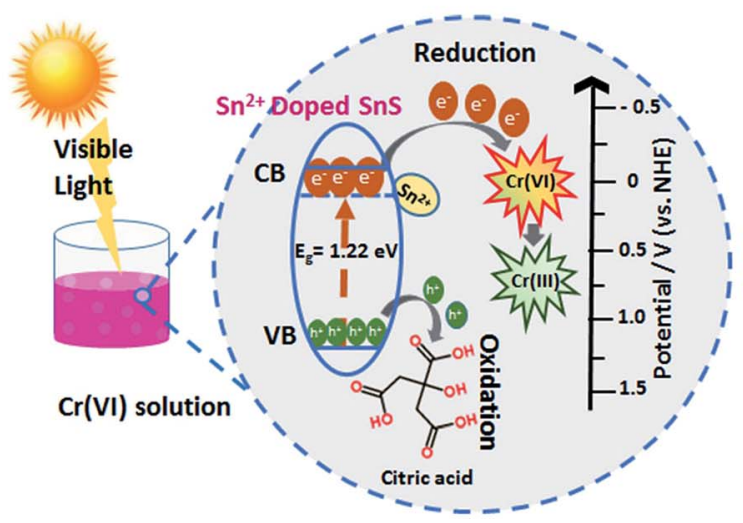

Fig. 12 (a) Mott-Schottky plots of $1: 1 \mathrm{SnS}$ and $1.1: 1 \mathrm{SnS}$ (b) energy band diagram of self-doped SnS.

effects of doping on the conduction band position and valence band position are described in Table 1. The valence band position was almost identical for all samples. However, the conduction band position changed; for undoped SnS, it was $-0.19 \mathrm{eV}$ ( $v s$. normal hydrogen electrode (NHE)), whereas for doped SnS (1.1: $1 \mathrm{SnS})$ it was $0.11 \mathrm{eV}(v s$. NHE), as shown in Table 1. Doping lowered the conduction band edge, consequently narrowing the band gap. In addition to this change, $\mathrm{Sn}^{2+}$ dopant sites formed as a result of doping, and these acted as trapping sites. For doped semiconductors, trapping sites increase the life time of charge carriers till an optimum point. This will eventually facilitate the reduction of $\mathrm{Cr}(\mathrm{vI})$. When the photocatalyst was irradiated, electrons travelled from the valence band to the conduction band, as shown in Fig. 12b, and then these electrons travelled from the conduction band of SnS and attacked $\mathrm{Cr}(\mathrm{VI})$, reducing it to $\mathrm{Cr}(\mathrm{III})$. The leftover holes generated in the valence band of doped SnS were consumed by citric acid. Overall, the $\mathrm{Sn}^{2+}$ self-doped samples were highly effective photocatalysts for the reduction of $\mathrm{Cr}(\mathrm{vI})$.

\section{Conclusions}

In the present study, $\mathrm{Sn}^{2+}$ self-doped $\mathrm{SnS}$ microspheres were synthesized by a simple hydrothermal method without using any template. We demonstrated that the non-stoichiometric self-doping scheme presented here is efficient and straightforward compared to external or impurity-based doping methods. The synthesized $\mathrm{Sn}^{2+}$ self-dopant $\mathrm{SnS}$ was used as a photocatalyst for the reduction of toxic $\mathrm{Cr}(\mathrm{vI})$ to $\mathrm{Cr}(\mathrm{III})$, and showed superior performance to undoped SnS and other reference photocatalysts. The $\mathrm{Sn}^{2+}$ self-dopant enhanced photocatalytic performance in two main ways. By creating an energy state near the conduction band of SnS, the self-dopant lowered the band gap of the parent material. The formation of intermediate energy state was also verified using Mott-Schottky method. Second, the $\mathrm{Sn}^{2+}$ self-dopant formed trap sites, minimizing the recombination problem. Improved photocatalytic reduction efficiencies obtained for the self-doped photocatalysts highlights the importance of self-doping in photocatalysts. In future studies, it is anticipated for exploring self-doped semiconductors as platforms for various electrochemical applications.

\section{Acknowledgements}

This work was supported by research grants of the NRF (2014M3A7B4052200, 2014M3C1A3053035) and Basic Science Research Program (2010-0027955) funded by the National Research Foundation under the Ministry of Science, ICT \& Future, Korea.

\section{Notes and references}

1 C. Karunakaran, S. Kalaivani and P. Vinayagamoorthy, Mater. Express, 2014, 4, 125-134.

2 L. Ma, L. Xu, X. Xu, X. Zhou and L. Zhang, NANO, 2016, 11, 1650035.

3 G. Liu, L. Wang, H. G. Yang, H.-M. Cheng and G. Q. (Max) Lu, J. Mater. Chem., 2010, 20, 831-843.

4 V. B. R. Boppana and R. F. Lobo, J. Catal., 2011, 281, 156-168.

5 M. Xing, J. Zhang, F. Chen and B. Tian, Chem. Commun., 2011, 47, 4947-4949.

6 M. Sun, Y. Su, C. Du, Q. Zhao and Z. Liu, RSC Adv., 2014, 4, 30820-30827.

7 H. Huang, X. Li, J. Wang, F. Dong, P. K. Chu, T. Zhang and Y. Zhang, ACS Catal., 2015, 5, 4094-4103.

8 F. Zuo, L. Wang, T. Wu, Z. Zhang, D. Borchardt and P. Feng, J. Am. Chem. Soc., 2010, 132, 11856-11857.

9 L. Chen, D. Meng, X. Wu, A. Wang, J. Wang, Y. Wang and M. Yu, J. Phys. Chem. C, 2016, 120, 18548-18559.

10 C.-M. Fan, Y. Peng, Q. Zhu, L. Lin, R.-X. Wang and A.-W. Xu, J. Phys. Chem. C, 2013, 117, 24157-24166.

11 A. Fujushima and K. Honda, Nature, 1972, 238, 37-38.

12 S. Chen and L. W. Wang, Chem. Mater., 2012, 24, 3659-3666.

13 A. Tanuševski and D. Poelman, Sol. Energy Mater. Sol. Cells, 2003, 80, 297-303.

14 J. Chao, Z. Wang, X. Xu, Q. Xiang, W. Song, G. Chen, J. Hu and D. Chen, RSC Adv., 2013, 3, 2746-2753.

15 K. T. Ramakrishna Reddy, N. Koteswara Reddy and R. W. Miles, Sol. Energy Mater. Sol. Cells, 2006, 90, 30413046 . 
16 J. J. Loferski, J. Appl. Phys., 1956, 27, 777-784.

17 P. Sinsermsuksakul, R. Chakraborty, S. B. Kim, S. M. Heald,

T. Buonassisi and R. G. Gordon, Chem. Mater., 2012, 24, 4556-4562.

18 D. Das and R. K. Dutta, J. Colloid Interface Sci., 2015, 457, 339-344.

19 Z. Fang, Q. Wang, X. Wang, F. Fan, C. Wang and X. Zhang, Mater. Res. Bull., 2013, 48, 4935-4941.

20 Y. Zhang, J. Lu, S. Shen, H. Xu and Q. Wang, Chem. Commun., 2011, 47, 5226-5228.

21 H. Li, T. Wu, B. Cai, W. Ma, Y. Sun, S. Gan, D. Han and L. Niu, Appl. Catal., B, 2015, 164, 344-351.

22 H.-T. Hsu, S.-S. Chen, W.-S. Chang and C.-W. Li, Desalin. Water Treat., 2013, 51, 495-502.

23 C. D. Pereira, J. G. Techy, E. M. Ganzarolli and S. P. Quináia, J. Environ. Monit., 2012, 14, 1559-1564.

24 F. Xie, X. Mao, C. Fan and Y. Wang, Mater. Sci. Semicond. Process., 2014, 27, 380-389.

25 A. Rauf, M. S. A. Sher Shah, G. H. Choi, U. Bin Humayoun, D. H. Yoon, J. W. Bae, J. Park, W.-J. Kim and P. J. Yoo, ACS Sustainable Chem. Eng., 2015, 3, 2847-2855.

26 V. M. Yuwono, N. D. Burrows, J. A. Soltis and R. L. Penn, J. Am. Chem. Soc., 2010, 132, 2163-2165.

27 X.-J. Dai, Y.-S. Luo, W.-D. Zhang and S.-Y. Fu, Dalton Trans., 2010, 39, 3426-3432.

28 K. M. Koczkur, S. Mourdikoudis, L. Polavarapu and S. E. Skrabalak, Dalton Trans., 2015, 44, 17883-17905.

29 W. Xia, H. Wang, X. Zeng, J. Han, J. Zhu, M. Zhou and S. Wu, CrystEngComm, 2014, 16, 6841-6847.

30 C.-H. Cheng, Y.-C. Chi, C.-L. Wu, C.-J. Lin, L.-H. Tsai, J.-H. Chang, M. K. Chen, M.-H. Shih, C.-K. Lee, C.-I. Wu, D. P. Tsai and G.-R. Lin, Nanoscale, 2016, 8, 4579-4587.
31 H. Wang, X. Lu, J. Tucek, J.-G. Zheng, G. Yang, R. Zboril and C. Niu, Electrochim. Acta, 2016, 211, 636-640.

32 M. Patel, A. Chavda, I. Mukhopadhyay, J. Kim and A. Ray, Nanoscale, 2016, 8, 2293-2303.

33 H. R. Chandrasekhar, R. G. Humphreys, U. Zwick and M. Cardona, Phys. Rev. B: Condens. Matter Mater. Phys., 1977, 15, 2177-2183.

$34 \mathrm{H}$. Kisch, Semiconductor Photocatalysis: Principles and Applications, Wiley-VCH verlag GmbH \& Co. KGaA, 2015.

35 A. Tauc, J. Grigorovici and R. Vancu, Mater. Res. Bull., 1966, 3, 37-46.

36 E. a. Davis and N. F. Mott, Philos. Mag., 1970, 22, 0903-0922.

37 S. State, O. Ridge and O. Ridge, Phys. Rev. B: Condens. Matter Mater. Phys., 1988, 37, 244-248.

38 N. Koteeswara Reddy and K. T. Ramakrishna Reddy, Mater. Chem. Phys., 2007, 102, 13-18.

39 B. Ghosh, M. Das, P. Banerjee and S. Das, Appl. Surf. Sci., 2008, 254, 6436-6440.

40 N. Sato, M. Ichimura, E. Arai and Y. Yamazaki, Sol. Energy Mater. Sol. Cells, 2005, 85, 153-165.

41 W. Choi, A. Termin and M. R. Hoffmann, J. Phys. Chem., 1994, 98, 13669-13679.

42 Q. Xie, H. Zhou, Z. Lv and H. Guo, J. Mater. Chem. A, 2017, 5, 6299-6309.

43 P. Mohapatra, S. K. Samantaray and K. Parida, J. Photochem. Photobiol., A, 2005, 170, 189-194.

44 J. M. Meichtry, M. Brusa, G. Mailhot, M. A. Grela and M. I. Litter, Appl. Catal., B, 2007, 71, 101-107.

45 D. Xu, B. Cheng, S. Cao and J. Yu, Appl. Catal., B, 2015, 164, 380-388. 